\title{
The 'PI 438489B' by 'Hamilton' SNP-Based Genetic Linkage Map of Soybean [Glycine max (L.) Merr.] Identified Quantitative Trait Loci that Underlie Seedling SDS Resistance
}

\author{
Kassem My Abdelmajid', Laura Ramos ${ }^{2}$, Leonor Leandro ${ }^{3}$, Gladys Mbofung ${ }^{3}$, David L. Hyten ${ }^{4}$, \\ Stella K. Kantartzi ${ }^{2}$, Robert L Grier IV ${ }^{1}$, Victor N. Niiti ${ }^{5}$, Silvia Cianzio ${ }^{3}$, and Khalid Meksem ${ }^{2 *}$ \\ 1 Plant Genomics \& Biotechnology Lab, Department of Biological Sciences, Fayetteville State University, Fay- \\ etteville, NC 28301, USA; ${ }^{2}$ Department of Plant, Soil, and Agricultural Systems, Southern Illinois University, \\ Carbondale, IL, USA; ${ }^{3}$ Plant Pathology Department and Agronomy Department, lowa State University, Ames, \\ IA 5001 1, USA; ${ }^{4}$ Soybean Genomics and Improvement Lab, 10300 Baltimore Ave, Bldg. 006, Rm. 201, Belts- \\ ville, MD 20705, USA; ${ }^{5}$ Department of Agriculture, Alcorn State University, Alcorn State, MS 39096.
}

Received: May 10, 2011 / Accepted: November 21, 2011

\begin{abstract}
Soybeans [Glycine $\max$ (L.) Merr.] are susceptible to many diseases including fungal diseases such as soybean sudden death syndrome (SDS). Several studies reported SDS resistance quantitative trait loci (QTL) on the soybean genome using different recombinant inbred line (RIL) populations and low density genetic linkage maps. High density exclusively single nucleotide polymorphisms-based (SNP-based) maps were not yet reported in soybean. The objectives of this study were (1) to construct a high density SNP-based genetic linkage map of soybean using the 'PI438489B' by 'Hamilton' (PIxH, $n=50)$ recombinant inbred line population, and (2) to map QTL for SDS resistance using this high-density reliable genetic SNP-based map. The PI438489B by Hamilton high-density SNP-based genetic map was a high density map composed of 31 LGs, 648 SNPs, and covered 1,524.7 cM with an average of $2.37 \mathrm{cM}$ between two adjacent SNP markers. Fourteen significant QTL were identified for SDS resistance using interval mapping (IM) and composite interval mapping (CIM) with LOD scores that ranged between 2.6 and 5.0. Twelve QTL were identified for foliar disease severity (FDS) and three QTL for root rot severity (RRS) of which one QTL underlain both FDS and RRS. The fourteen QTL were mapped onto ten separate chromosomes of the soybean genome. Seven of the intervals encompassing the QTL had been identified previously (on LGs C1, C2, D1 b, G, L, N and O) associated
\end{abstract}

with resistance to SDS but seven were novel (LGs A2 (2), B1, C2, D1 a, D1 b and O). We constructed the first P1438489B by Hamilton exclusively SNP-Based map and identified fourteen QTL that underlie SDS resistance including both resistances to foliar and root rot symptoms caused by Fusarium virguliforme infection. The QTL discovered here for SDS resistance could be useful to include in breeding programs in developing soybean cultivars resistant to SDS.

\section{Introduction}

Soybean [Glycine max (L.) Merr.] sudden death syndrome (SDS) is caused by the fungus Fusarium virguliforme (Aoki et al., 2005). SDS is capable of causing major yield losses in soybean (Luo et al., 2000). Host resistance in soybean to SDS has been found to be polygenic and controlled by several quantitative trait loci (QTL) (Meksem et al., 1999; lqbal et al., 2001).

In the 'Essex' by 'Forrest' recombinant inbred line (RIL) population ( $E x F, n=100)$ nine QTL have been discovered (Kassem et al., 2006, 2007). More specifically, two were found on chromosome 13 (Linkage Group [LG]) F); one QTL each on chromosomes 16 (LG J), 6 (LG C2), and 20 (LG I); and four QTL on chromosome 18 (LG G) (Kassem et al., 2007). To date 3 of these QTL were confirmed in near isogeninic lines (NILs); two on LG 
$G$ and one on LGC2. Three additional QTL for SDS resistance have been found on chromosomes 3 (LG N), 6 (LG C2), and 18 (LG G) using the 'Pyramid' by 'Douglas' RIL population (PxD, $n=90$ ) (Kassem et al., 2007; Niiti et al., 2002). Prabhu et al. (1999) tested the markers Satt038 (chromosome 18 - LG G) and BLT65 (chromosome 8 - LG A2) and found QTL for SDS resistance associated with the LG G markers in the 'Hartwig' by 'Flyer' RIL population ( $F x H, n=50)$ (Prabhu et al., 1999). Farias et al. (2007) mapped QTL for SDS resistance (DX) using two RIL populations 'Ripley' by 'Spencer' (RxS, n=91) and PI 567374 by 'Omaha' (PI567374xO, n=96) (Farias et al. 2007). These QTL were located on chromosomes 4, 17, and 19 (LGs C1, D2, and $L$, respectively) in the first population and on chromosomes 17 and 20 (LGs D2 and I) in the latter one (Farias et al., 2007). It is worth mentioning thatthe D2 QTL was confirmed in NILs too. In a recent study, Kazi et al. (2007) identified QTL on LG C2, D2 and $\mathrm{G}$ in $\mathrm{FxH}(\mathrm{n}=94)$ and confirmed the D2 QTL in NILs (Kazi ef al., 2007; Kazi et al., 2008). However, the ExF, HxF, PxD, RxS, and $\mathrm{PI} 567374 \times \mathrm{O}$ genetic linkage maps that were used to map SDS resistance QTL were not high density genetic maps and also the populations that used were limited. The ExF genetic linkage map was based on 237 markers (Kassem et al., 2006), the FxH genetic linkage map was composed of 144 markers (Kazi et al., 2007, 2008), the PxD genetic linkage map was composed of 112 markers (Njiti et al., 2002), the RxS map was composed of 112 simple sequence repeats (SSRs) with only 68 of the markers linked (Farias et al. 2007), and the PI567374xO map was composed of 104 SSRs with only 41 of the markers linked (Farias et al., 2007).

During the last two decades, soybean genetic maps have been increasing in the number of markers used to create the maps. The first genetic map in soybean was based on 150 markers (Keim et al., 1990). Subsequently, the number of markers used to create genetic maps has varied greatly and many different types of markers such as restriction fragment length polymorphisms (RFLPs), amplified fragment length polymorphisms (AFLPs), random amplifications of polymorphic DNA (RAPDs), and SSRs have used.. The number of markers has varied from 110 markers (Shoemaker et al., 1995), to 112 markers (Niiti et al., 2002), to 132 markers (Lark et al., 1995), to 237 markers (Kassem et al., 2006), to high density composite genetic maps (Song et al., 2004).

Since the discovery of single nucleotide polymorphism (SNP) markers in mice and humans (Collins et al., 1998; Lindblad-Toh et al., 2002), several methods of SNP discovery have been described in different species including plants (Nicod et al., 2003; Barbazuk et al., 2007; Hyten et al., 2010a). SNPs are the most abundant genetic marker available for the creation of genetic maps and can be used with high-throughput genotyping technology making them highly desirable for QTL mapping studies (Brookes et al., 1999; Nicod et al., 2003; Barbazuk et al., 2007; Hsu et al., 2008; Fan et al., 2003). On this basis, SNPbased maps have been constructed in many plant species including Arabidopsis thaliana (Li et al., 2000), almond (Wu et al., 2009), sugar beet (Mohring et al., 2004), soybean (Hyten et al., $2010 b)$, and others. However, the genetic maps in soybean have not been exclusively SNP-based but contain SNPs anchored with other markers such as RFLPs, AFLPs, RAPDs, and SSRs except of a recent high density soybean SNP-based map containing 1,790 SNPs (Hyten et al., 2010a).

Genetic linkage maps have been used to identify and map QTL for many important agronomic traits in soybean (SoyBase, 2011 ). High density maps, the marker-type, and population size, are among important factors for mapping QTL. The objectives of this study were (1) to construct a high density SNP-based genetic linkage map of soybean using the PI 438489B by ' $\mathrm{Ham}$ ilton' (PIxH, n=50) RIL population, and (2) to map QTL for SDS resistance using this high-density genetic SNP-based map.

\section{Materials and Methods}

\section{Plant Material}

In this study, we used the PI $438489 B$ by Hamilton recombinant inbred line (RIL) population ( $\mathrm{PIxH}, \mathrm{n}=50$ ). $\mathrm{PI} 438489 \mathrm{~B}$ is a plant introduction from China and is resistant to SDS. Hamilton was developed at the Illinois Agricultural Experiment Station and was released for its high yield (Nickell et al., 1990) although it is susceptible to SDS. The PI $438489 \mathrm{~B}$ by Hamilton RIL population was developed at the University of Missouri Agronomy Research Center (Yue et al., 2001) and advanced to the $F_{6: 13}$ generation by Dr. Silvia Cianzio at the ISU research site at the Isabela Substation, Univ. of Puerto Rico, Isabela, Puerto Rico.

\section{DNA Isolation}

DNA was extracted using DNeasy 96 Tissue Kit (QIAGEN, Inc., Valencia, CA, USA) from young leaf tissue. DNA quantification was performed using fluorescent nucleic acid stain with Hoechst 33258 dye (Cat no. H21491 Invitrogen, Carlsbad, CA, USA). The DNA samples were read in a Synergy 2 plate reader

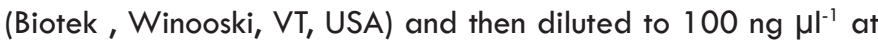
final concentration.

\section{SNP Genotyping}

The 1,536 Universal Soy Linkage Panel 1.0 from Hyten et al. (2010b) was used to screen the 50 RILs and the GoldenGate assay was performed as per the manufactures protocols and as described previously (Fan et al., 2003; Hyten et al., 2008). The Illumina BeadStation 500G (Illumina Inc., San Diego, CA) was used for genotyping the GoldenGate assay. The automatic allele calling for each locus was accomplished with the BeadStudio version 3.2 software (Illumina Inc., San Diego, CA). All BeadStudio data for the 1,536 SNPs were visually inspected and rescored if any errors in calling the homozygous or heterozygous clusters were evident.

\section{SDS Phenotypic Scoring}

The RIL population was phenotyped for disease severity in the plant pathology greenhouse at lowa State University, in Ames, lowa. The experiment was established as a randomized complete block design with three replications per RIL. The 
experimental unit was a 3" styrofoam cup containing three plants growing in soil infested with $F$. virguliforme. Inoculum was prepared according to a protocol by Munkvold and O'Mara (2002). One kilogram of a sterile 5:1 sand:cornmeal mixture was infested with $0.6 \mathrm{ml}$ of a 106 spore $/ \mathrm{ml}$ suspension of the Mont 1 isolate of F. virguliforme (Li et al., 2000), resulting in an initial spore concentration of 600 spores $\mathrm{g}^{-1}$ of inoculum. The inoculum was incubated for 10 days at room temperature (approximately $24^{\circ} \mathrm{C}$ ), after which it was homogeneously mixed in a 1:5 ratio with pasteurized soil and sand $\operatorname{mix}(1: 1$ by vol). Three seeds were then planted in styrofoam cup filled with the infested soil mix. The cups were maintained on a greenhouse bench at $23 \pm 2{ }^{\circ} \mathrm{C}$, and a $16 \mathrm{~h}$ photoperiod for 30 days. Foliar diseases severity (FDS) was rated 15 (FDS 1), 20 (FDS 2), 25 (FDS 3), and 30 (FDS 4) days after planting (DAP) as a percentage of total leaf area with typical SDS symptoms. Root rot severity (RRS) was evaluated 30 DAP by thoroughly washing the roots in running tap water and visually evaluating the percentage of root area showing brown or black discoloration. This highly controlled assay has been shown to produce results in seedlings that correlate closely with field performances (Niiti, 2001). The experiment was conducted twice.

\section{Statistical Data Analysis}

Means, ranges and standard deviations were calculated for the RIL lines and their parents from raw data. Analyses were performed on JMP 8.02 (SAS Institute Inc., Cary, NC, USA).

\section{QTL Mapping}

For mapping of QTL and estimation of their effects, composite interval mapping ( $\mathrm{CIM})$ was performed using the WinQTL Cartographer version 2.5 software (Wang et al., 2005). The Model 6 and its default settings were adopted. To establish experimental-wise LOD cutoff values for declaring QTL significant at $\mathrm{P} \leq 0.051,000$ permutations were performed. In addition, a value of $P \leq 0.0002$ or LOD 3.7 was suggested by an approximate Bonferroni correction $(P<0.05 / 250)$ for the set of about 250 independent (unlinked or $>10 \mathrm{cM}$ apart) DNA markers (from the 679 mapped). However, at genomic regions where gaps between adjacent markers were greater than $10 \mathrm{cM}$ in the map associations $0.005>P>0.0005(2.3<$ LOD $<3.3)$ were accepted as a potentially significant association. If the interval was large or was flanking a single marker the uncorrected $P$ value of $<0.05$ was accepted. Precedents with first-pass mapping of other quantitative traits (lqbal et al., 2001; Kassem et al., 2006, 2007; Njiti et al., 2002; Prabhu et al., 1999; Farias et al., 2007; Kazi et al., 2007) have shown these criteria to be valid during the later NIL based maps of the intervals that were inferred at marginal $P$ values (Meksem et al., 1999).

\section{Genetic Map Construction}

The current PI 438489B by Hamilton genetic map is exclusively SNP-based and was constructed in several steps using JoinMap 4.0 software (Feltus et al., 2010). The SNP markers were initially grouped and assigned to the soybean chromosomes based on their mapped position on the soybean Consensus 4.0 map (Hyten et al., 2010b). We used the regression mapping algorithm with the default parameters and Kosambi's mapping function to determine map order and genetic distances.

\section{Finding Potential Genes that Underlie SDS QTL}

Each QTL was bounded by two single nucleotide polymorphisms (SNPs). The DNA sequence of the SNPs was obtained by searching the NCBI Single Nucleotide Polymorphism database (http://www.ncbi.nlm.nih.gov/projects/SNP/). The SNP DNA sequences were used as a query in a BLAST search of the Glycine max genome, version Williams 82 genomic sequence (http:// soybase.org/gbrowse/cgi-bin/gbrowse/gmax 1.01/). The BLAST search revealed the nucleotide position of each SNP on the chromosome of interest. The nucleotide positions were used to query the soybean whole genome sequence (Glycine max version 1.01) (Schmutz et al., 2010) that included gene names and gene descriptions (http://soybase.org/gbrowse/cgi-bin/ gbrowse/gmax 1.01/). The results included the genes and gene descriptions of all genes bounded by the SNPs, and the data was pasted into Microsoft Excel version 2007 spreadsheet for subsequent analysis. To determine common genes within all QTL intervals, a single excel file was created with three columns: QTL, gene name and gene description. The QTL and gene name column were concatenated to form the QTL/gene column and a delimiter (semicolon) was appended at the end. The QTL and gene name columns were deleted leaving two columns: QTL/gene and gene description. The excel function Remove Duplicates was used to remove duplicate QTL/genes in a given QTL based on the gene description. The columns were sorted alphabetically based on gene description. The following function identified genes that were common to more than one QTL and created a new column $(=\mathrm{IF}(\mathrm{OR}(\mathrm{B} 2=\mathrm{B} 3, \mathrm{~B} 2=\mathrm{B} 1), \mathrm{A} 2$ \&" "\& B2) where $\mathrm{A}$ is the $\mathrm{QTL} /$ gene column and $B$ is the gene description column. Genes that occurred only in one QTL were labeled as false. The column was sorted alphabetically and the false values deleted. The excel function Text to columns was used to separate the column into two columns using the semicolon delimiter. The first column was the QTL/gene data and the second column was the gene description. The columns were sorted alphabetically based on the gene description column. The function (=COUNTIF $A: A, A 1$ ) where $A$ is the column with gene description was used to count the number of repeating gene descriptions, and a new column gene frequency was created. The three columns QTL/gene, gene description and gene frequency were sorted based on gene frequency. The results are presented in Table 4 (Supplementary Data).

\section{Results}

\section{Trait Distributions}

SDS resistance was determined in the RIL population using foliar disease severity (FDS) and root rot severity (RRS) which are symptoms caused by $F$. virguliforme infection of plants in 
the greenhouse. FDS 1, 2, 3, and 4 were scored 15, 20, 25, and 30 days after planting (DAP), respectively and RRS was estimated 30 DAP. Means and standard deviations of FDS 1-4 and RRS are shown in Table 1. The RIL population demonstrated a broad range of variation for the FDS and RRS traits that were evaluated (Table 1). The variation of FDS 1 ranged from 0 to 30 (st. dev. 2.437), FDS 2-4 ranged from 0-100 (st. dev 31.18935.929 ) and RRS showed range from 0 to 100 (st. dev. 14.255) (Table 1).

Table 1. Means, ranges, skewness and test for normal distribution within the PI438489B by Hamilton RIL population.

\begin{tabular}{lcc}
\hline Traits & Mean $( \pm$ St. Dev. $)$ & Range \\
FDS 1 & $0.406( \pm 2.437)$ & $0-30$ \\
FDS 2 & $17.461( \pm 31.189)$ & $0-100$ \\
FDS 3 & $47.677( \pm 35.929)$ & $0-100$ \\
FDS 4 & $62.239( \pm 32.450)$ & $0-100$ \\
RRS & $89.072( \pm 14.255)$ & $0-100$ \\
\hline
\end{tabular}

\section{SNP-Based Genetic Map}

The PI 438489B by Hamilton SNP-based genetic map was constructed in several steps using the JoinMap 4.0 software (Feltus et al. 2010). First, the 1536 SNP Universal Soy Linkage Panel 1.0 from Hyten et al. (2010b) was used to screen the parents PI 438489B and Hamilton, and the 50 RILs. Out of the 1,536 SNPs there were a total of 679 SNPs which were polymorphic between the two parents and segregated within the RIL population. These 679 SNPs were used to construct the genetic linkage map. The final genetic map contained 31 LGs and 648 linked SNP markers (Figure 1). Thirty-one markers were unlinked. The map coverage was 1,524.7 cM (Figure 1). The average distance between markers was $2.35 \mathrm{cM}$ (Table 3 ). This map was used for genetic mapping of the SDS resistance QTL.

\section{SDS Resistance QTL}

Both interval mapping (IM) and composite interval mapping (CIM) were used to identify SDS resistance QTL. Using CIM, eleven QTL were identified for SDS resistance and mapped on 10 separate chromosomes of the soybean genome. Eight QTL

Table 2. The fourteen QTL that underlie SDS resistance found in the soybean PI 438489B by Hamilton RIL population. The QTL were found using the CIM of WinQTL Cart., and reported with LOD scores equal or greater than 2.5. Traits measured were foliar disease severity (FDS 1, 2, 3, or 4 weeks after infection by F. virguliform, respectively) and root rot severity (RRS) symptoms. QTL were named according to the Soybean Genetics Committee recommendations as revised in March 2007 (http://soybase.org/resources/QTL.php).

\begin{tabular}{|c|c|c|c|c|c|c|c|}
\hline Trait & No. & QTL & Chr./LG & Marker/Interval & Position (cM) & LOD & $\overline{\mathbf{R}^{2}(\%)}$ \\
\hline \multicolumn{8}{|l|}{ CIM } \\
\hline \multirow[t]{3}{*}{ FDS 2} & 1 & qFDS002-01 & $1 / D 1 a$ & ss107927723-ss107913849 & $27.2-42.8$ & 3.0 & 7.5 \\
\hline & 2 & qFDSO02-02 & $10 / 0$ & ss107930838-ss107912519 & $13.5-15.2$ & 2.6 & 19.3 \\
\hline & 3 & qFDS002-03 & $19 / \mathrm{L}$ & ss107913933-ss107929955 & $42.0-49.9$ & 4.0 & 17.7 \\
\hline \multirow[t]{4}{*}{ FDS 3} & 4 & qFDS003-01 & $2 / D 1 b$ & ss107927695-ss107913858 & $19.4-21.8$ & 3.6 & 9.0 \\
\hline & 5 & qFDS003-02 & $2 / \mathrm{D} 1 \mathrm{~b}$ & ss107920774-ss107912689 & $30.0-36.0$ & 2.8 & 5.2 \\
\hline & 6 & qFDS003-03 & $6 / C 2$ & ss107929602-ss1 07925487 & $32.8-39.2$ & 4.2 & 3.5 \\
\hline & 7 & qFDS003-04 & $8 / \mathrm{A} 2$ & ss107919498-ss107915722 & $2.0-13.0$ & 4.6 & 9.6 \\
\hline \multirow[t]{2}{*}{ FDS 4} & 8 & qFDS004-01 & $11 \mathrm{~b} / \mathrm{B} 1$ & ss $107912672-s s 107924081$ & $5.5-17.8$ & 2.7 & 3.4 \\
\hline & 9 & qFDS004-02 & $18 / G$ & ss4969823-ss107924619 & $24.4-28.1$ & 2.9 & 8.8 \\
\hline \multirow[t]{3}{*}{ RRS } & 10 & qRRS001-01 & $3 / N$ & ss $107912585-s s 107920575$ & $38.3-42.6$ & 4.2 & 9.9 \\
\hline & 11 & qRRS001-02 & $4 / \mathrm{Cl}$ & ss107929213-ss107929551 & $51.5-57.3$ & 5.0 & 8.6 \\
\hline & (9) & qRRS001-03 & $18 / G$ & ss4969823-ss107924619 & $24.4-27.7$ & 2.9 & 33.3 \\
\hline \multicolumn{8}{|l|}{ IM } \\
\hline FDS 2 & (3) & qFDS002-03 & $19 / \mathrm{L}$ & ss107924889-ss107921208 & $48.0-51.3$ & 2.6 & 6.0 \\
\hline \multirow[t]{3}{*}{ FDS 3} & 12 & qFDS003-05 & $6 / C 2$ & ss107917031-ss107912977 & $16.9-32.8$ & 4.2 & 2.4 \\
\hline & (6) & qFDS003-03 & $6 / C 2$ & ss107930961-ss107912561 & $34.5-39.8$ & 4.6 & 4.7 \\
\hline & 13 & qFDS003-06 & $8 / \mathrm{A} 2$ & ss107915722-ss107918074 & $15.0-28.0$ & 2.7 & 17.4 \\
\hline \multirow[t]{3}{*}{ FDS 4} & 14 & qFDS004-03 & $4 / \mathrm{Cl}$ & ss107924445-ss107918378 & $57.3-83.9$ & 2.6 & 4.8 \\
\hline & $(10)$ & qFDS004-03 & $6 / C 2$ & ss107917031-ss107912977 & $16.9-32.8$ & 3.4 & 2.1 \\
\hline & (6) & qFDS004-04 & $6 / C 2$ & ss107930961-ss107912561 & $34.5-39.8$ & 3.6 & 3.2 \\
\hline RRS & $(3-9)$ & qRRS001-03 & $18 / G$ & ss107924669-ss107921695 & $26.5-28.4$ & 2.6 & 2.3 \\
\hline
\end{tabular}


Table 3. Statistics of the linkage groups (LGs) of the 'PI 438489B' by 'Hamilton' SNP-based genetic linkage map.

\begin{tabular}{|c|c|c|c|}
\hline LG. Chr. No & $\begin{array}{l}\text { Coverage } \\
\text { (cM) }\end{array}$ & $\begin{array}{l}\text { No. of } \\
\text { Markers }\end{array}$ & cM/Marke \\
\hline 1. Chr_1 & 93.0 & 32 & 2.91 \\
\hline 2. Chr_2 & 60.2 & 31 & 1.94 \\
\hline 3. Chr_3 & 51.7 & 24 & 2.15 \\
\hline 4. Chr_4 & 98.4 & 31 & 3.17 \\
\hline 5. Chr_5a & 49.8 & 18 & 2.76 \\
\hline 6. Chr_5b & 34.1 & 8 & 4.26 \\
\hline 7. Chr_6 & 68.4 & 44 & 1.55 \\
\hline 8. Chr_7 & 79.9 & 35 & 2.28 \\
\hline 9. Chr_8 & 119.4 & 50 & 2.39 \\
\hline 10. Chr_9 & 73.0 & 53 & 1.38 \\
\hline 11.Chr_10 & 118.5 & 26 & 4.55 \\
\hline 12.Chr_11a & 4.5 & 12 & 0.37 \\
\hline 13.Chr_11b & 18.7 & 8 & 2.34 \\
\hline 14.Chr_11c & 18.2 & 4 & 4.55 \\
\hline 15.Chr_11d & 6.9 & 4 & 1.72 \\
\hline 16. Chr_12 & 58.5 & 25 & 2.34 \\
\hline 17.Chr_13a & 35.0 & 18 & 1.94 \\
\hline 18.Chr_13b & 65.9 & 21 & 3.14 \\
\hline 19. Chr_14 & 99.5 & 33 & 3.01 \\
\hline 20.Chr_15a & 37.1 & 10 & 3.71 \\
\hline 21.Chr_15b & 37.3 & 25 & 1.49 \\
\hline 22. Chr_16a & 28.2 & 7 & 4.03 \\
\hline 23. Chr_16b & 37.9 & 12 & 3.16 \\
\hline 24. Chr_17a & 23.8 & 16 & 1.48 \\
\hline 25.Chr_17b & 27.7 & 8 & 3.46 \\
\hline 26.Chr_17c & 16.8 & 15 & 1.12 \\
\hline 27.Chr_17d & 11.9 & 3 & 3.96 \\
\hline 28. Chr_18 & 35.1 & 30 & 1.17 \\
\hline 29.Chr_19 & 54.2 & 16 & 3.38 \\
\hline 30. Chr_20a & 22.5 & 12 & 1.87 \\
\hline 31.Chr_20b & 38.6 & 11 & 3.51 \\
\hline Total & 1524.7 & 642 & 2.37 \\
\hline
\end{tabular}

were found for foliar (FDS) resistance only, two QTL for root rot (RRS) resistance only, and one QTL which conferred resistance on both leaves and roots. The first QTL was associated with the FDS 2 trait (qFDS002-01) was identified in the marker interval ss 107927723-ss107913849 on chromosome 1 (LG Dla). The QTL interval spanned approximately $15.6 \mathrm{cM}$, had a peak LOD score of 3.0, and an R2 of 7.5\% (Table 2, Figure 1). The second QTL associated with the FDS 2 trait (qFDSO02-02) was identified in the marker interval ss 107930838-ss107912519 on chromosome 10 (LG O). The QTL interval spanned approximately $1.7 \mathrm{cM}$, had a peak LOD score of 2.6 , and an R2 of $19.3 \%$ (Table 2, Figure 1). The third QTL which was associated with the FDS 2 trait (qFDSO02-03) was identified by the marker interval ss107913933-ss107929955 on chromosome 19 (LG L). The QTL interval spanned approximately $7.9 \mathrm{cM}$, had a peak LOD score of 4.0 , and an R2 of $17.7 \%$ (Table 2, Figure 1). The three QTL explained approximately $50 \%$ of the total variation in FDS 2. The fourth QTL which was associated with the FDS 3 trait (qFDS003-01) was identified by the marker interval ss 107927695-ss107913858 on chromosome 2 (LG D1b). The QTL interval spanned approximately $2.4 \mathrm{cM}$, had a peak LOD score of 3.6, and an R2 of 9.0\% (Table 2, Figure 1). The fifth QTL that was associated with the FDS 3 trait (qFDS003-02) was identified by the marker interval ss 107920774-ss107912689 on the same chromosome 2 (LG DIb). The QTL interval spanned approximately $6 \mathrm{cM}$, had a peak LOD score of 2.8 , and an R2 of $5.2 \%$ (Table 2, Figure 1). The sixth QTL that was associated with the FDS 3 trait (qFDS003-03) was identified by the marker interval ss $107929602-$-ss 107925487 on chromosome 6 (LG C2). The QTL interval spanned approximately $6.4 \mathrm{cM}$, had a peak LOD score of 4.2 , and an R2 of 3.5\% (Table 2, Figure 1). The seventh QTL that was associated with the FDS 3 trait (qFDS003-04) was identified by the marker interval ss 107919498-ss107915722 on the same chromosome 8 (LG A2). The QTL interval spanned approximately $11 \mathrm{cM}$, had a peak LOD score of 4.6 , and an R2 of $9.6 \%$ (Table 2, Figure 1). The four QTL explained approximately $43.7 \%$ of the total variation in FDS 3 . The eighth QTL that was associated with the FDS 4 trait (qFDS004-01) was identified by the marker interval ss $107912672-$ ss 107924081 on chromosome 11 (LG B1). The QTL interval spanned approximately $12.3 \mathrm{cM}$, had a peak LOD score of 2.7 , and an R2 of $3.4 \%$ (Table 2, Figure 1). The ninth QTL that was associated with the FDS 4 trait (qFDS004-02) was identified by the marker interval ss4969823-ss107924619 on chromosome 18 (LG G). The QTL interval spanned approximately $3.7 \mathrm{cM}$, had a peak LOD score of 2.9, and an R2 of $8.8 \%$ (Table 2, Figure 1). This same interval which was significantly associated with FDS 4 also was significant for RRS (qRRSO0 1-03). The QTL interval spanned approximately $3.3 \mathrm{cM}$, had a peak LOD score of 2.9 , and an R2 of $33.3 \%$ (Table 2, Figure 1). The two QTL explained approximately $26.4 \%$ of the total variation in FDS 4 . The tenth QTL that was associated with the RRS trait (qRRS001-01) was identified by the marker interval ss $107912585-$ ss107920575 on chromosome 3 (LG N). The QTL interval spanned approximately $4.3 \mathrm{cM}$, had a peak LOD score of 4.2 , and an R2 of $9.9 \%$ (Table 2, Figure 1). The eleventh QTL that was associated with the RRS trait (qRRS001-02) was identified by the marker interval ss 107929213-ss107929551 on chromosome 4 (LG C1). The QTL interval spanned approximately $5.8 \mathrm{cM}$, had a peak LOD score of 5.0, and an R2 of $8.6 \%$ (Table 3, Figure 1). Another QTL associated with the RRS trait (qRRS001-03) was identified by the marker interval ss4969823-ss107924619 on chromosome 18 (LG G). The QTL interval spanned approximately 3.3 $\mathrm{CM}$, had a peak LOD score of 2.9, and an R2 of 33.3\% (Table 2, Figure 1). However, this QTL is the same as qFDS004-02 described above. The three QTL explained approximately $53.5 \%$ of the total variation in RRS.

Using IM, three additional QTL were identified for SDS foliar resistance (FDS). The twelfth QTL that was associated with the FDS 3 trait (qFDS003-05) was identified by the marker interval 


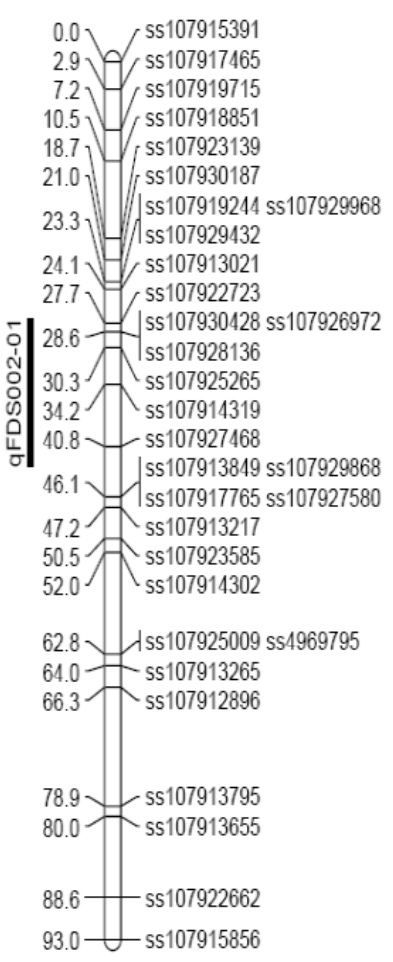

Chr_5a

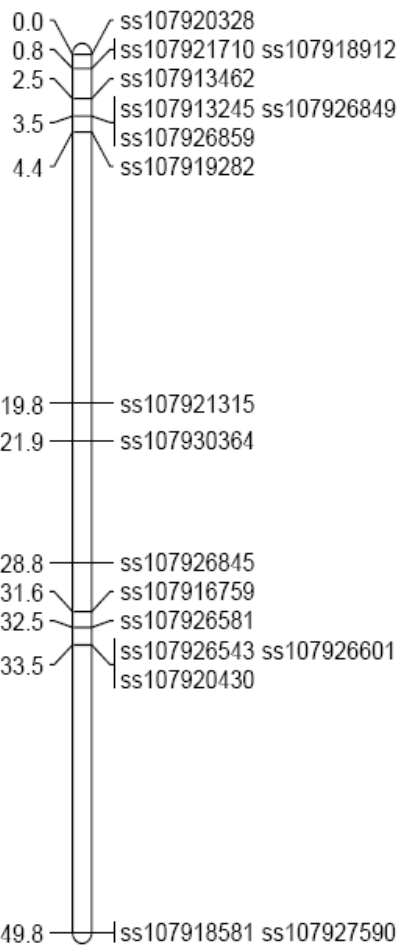

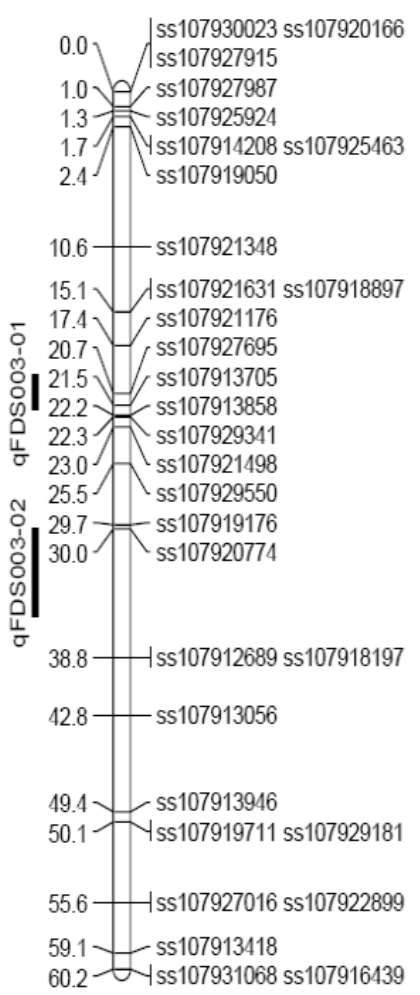

$$
\text { Chr_5b }
$$

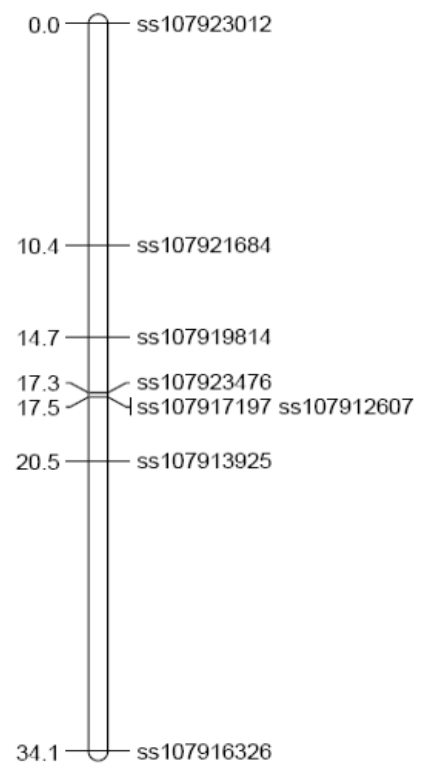

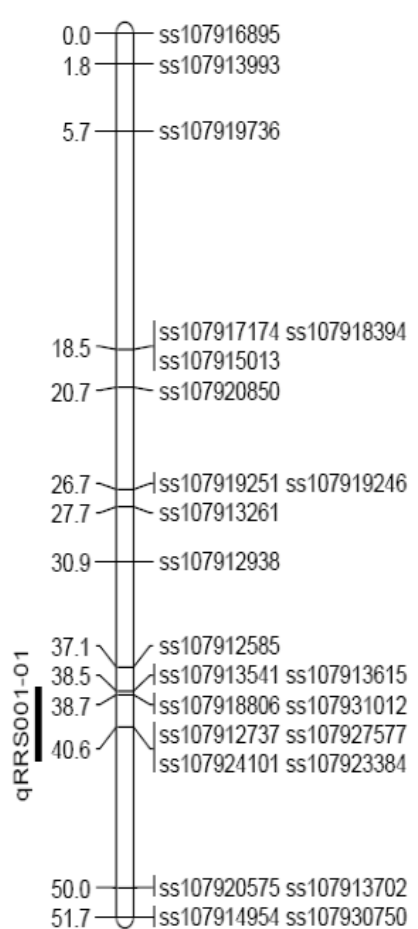

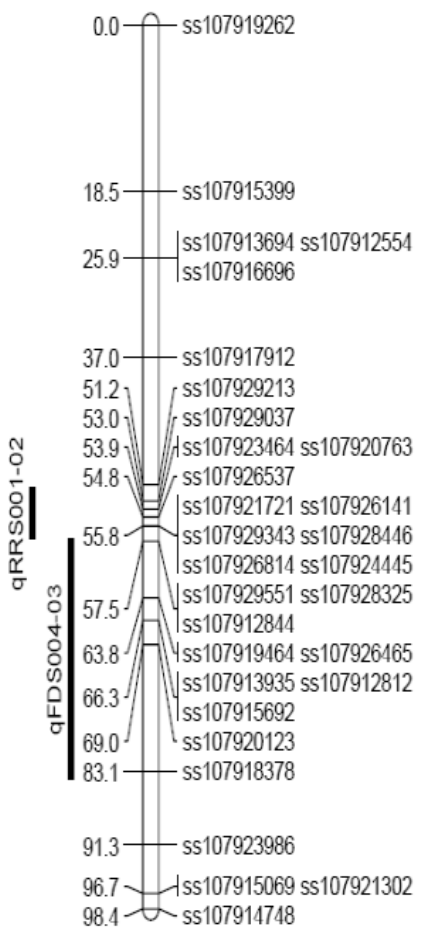

Chr_7

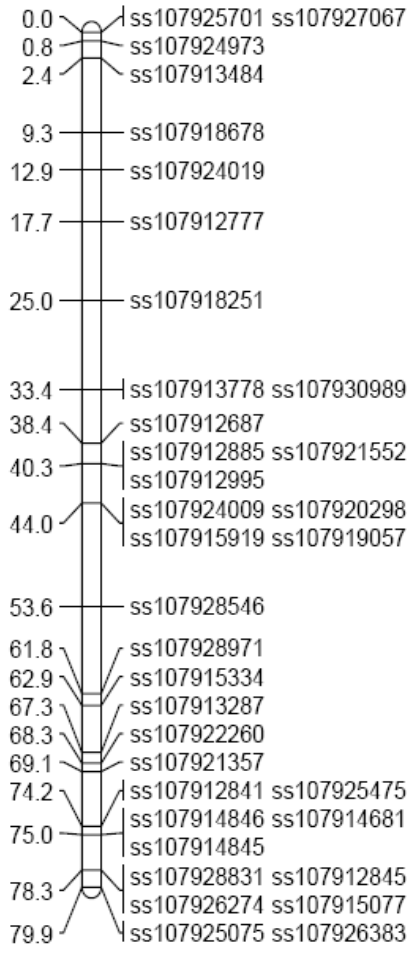

Figure 1. The 'PI 438489B' by 'Hamilton' SNP-based genetic linkage map and the positions of the fifteen QTL that underlie SDS resistance found in this RIL population $(n=50)$. 
Chr_9

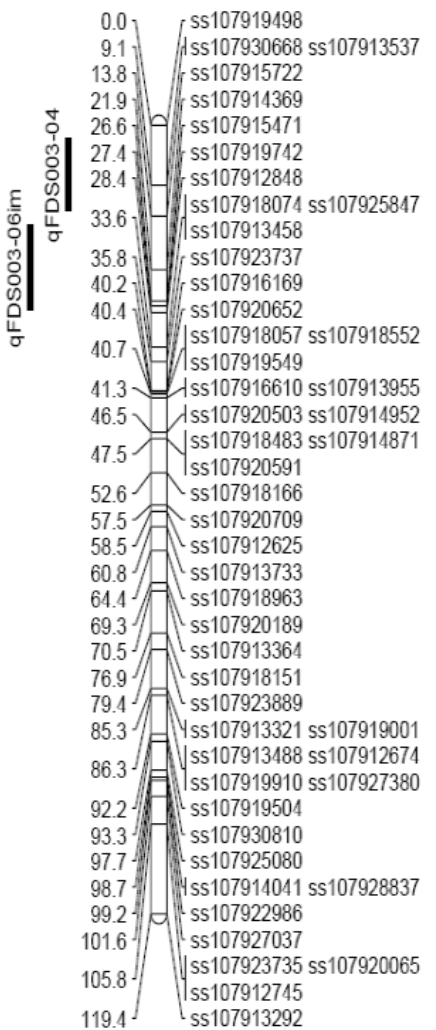

Chr_11b
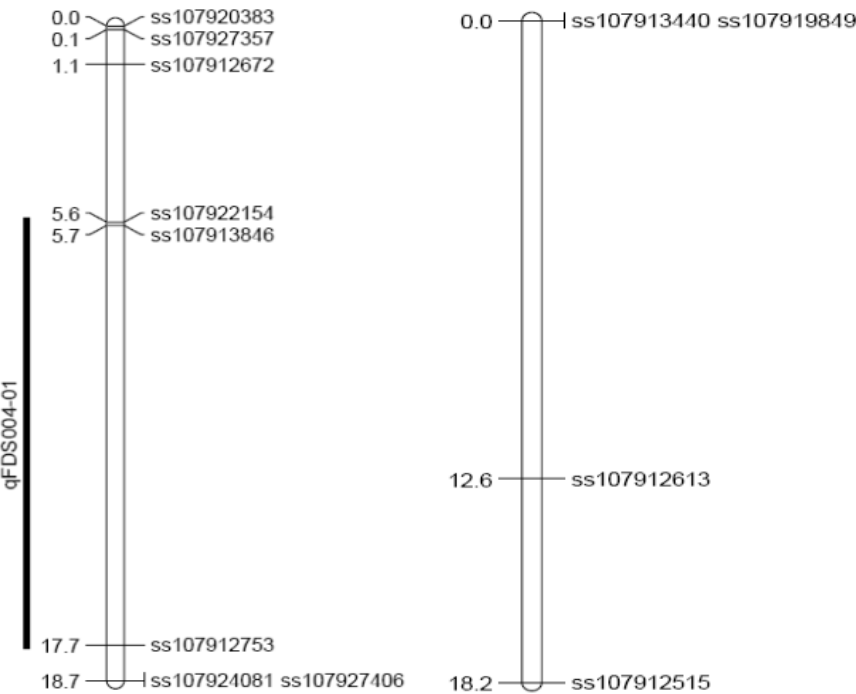

0.0 Iss107912559 ss107913820

6.0 $\mathrm{ss} 107922869$

7.0 - $\mathrm{s}$ s 107917952

9.1) $\$ s 107927867$

136. $\$$ \$s 107922775

16.8 . d ss107913799 ss107921298

23.7 ss107924405

24.8 - ss107918132

25.7 | ss107913779 ss107912826

- I Is 107930335

30.5] ss107914945

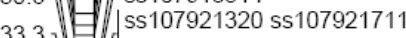

33.3 ) $/$ ss107928760

33.4 dss107913464 ss107921583

ss107926758 ss107925839

33. 作 ss107928776 ss107926331 ss107925455 ss107912644 ss107913900 ss107923621 ss107917237

34.1 - Iss107922884 ss107918158 34.5 -

ss107924441

35.0 ss107924569

37.9 - -107924931

39.0 Mss107913002 ss107915686

44.6

15.3 A $\mathrm{As} 4969766$

48.1

53.2 ss 107923267

59.5 ]

60.6 ss107918120

62.4 A 4 ss107930580 ss107913760

65.0

69.3 ss107922655

$\left.\begin{array}{l}72.1 \\ 73.0\end{array}\right] \quad\left[\begin{array}{l}\text { ss } 107923059 \\ s s 107921581\end{array}\right.$

Chr_11c
Chr_10

Chr_11a

$\left.\begin{array}{l}1.0 \\ 51\end{array}\right]$ Ss $\begin{aligned} & 5107924298 \\ & \text { ss } 107912624 \\ & \text { ss } 107927145\end{aligned}$

$30.6]$ ss107913314

ํํㅇ

ำ 15.8 / ss107930838

I 16.4 iss107912519ss107921621

م 17.3 Yss107925846 ss107921014

o 19.2 ss4969617

21.8 Yss107914627 ss107913214

42.2) ss107912744

44.2 ss107920438

45.2 $\sqrt{ }$ ss4969789

$47.4=\mathrm{ss} 107921648$

48.5 Ss 107925732

52.1 - ss107913294

54.4 ss107918469

55.7 ss107930848

70.7 ss107923829

72.8 - Iss107926354 ss107915512

$73.7 \gamma_{\mathrm{ss} 107927744}$

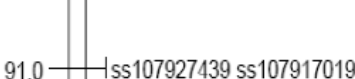

$95.7-$ ss 107922716

$99.5-$ - ss107915086

$118.5 \mathcal{J}_{\mathrm{ss} 107929403}$

Chr_11d

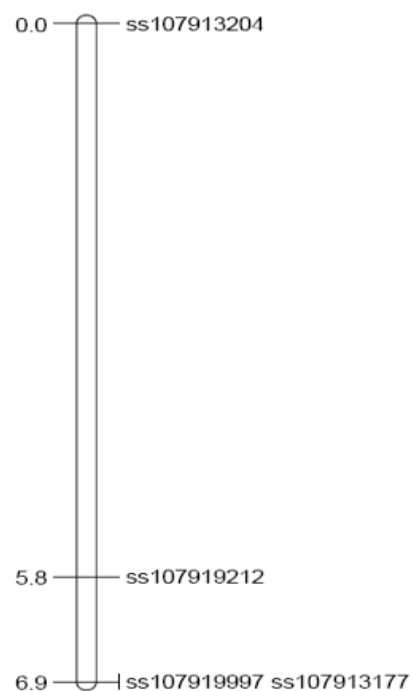

Chr_12
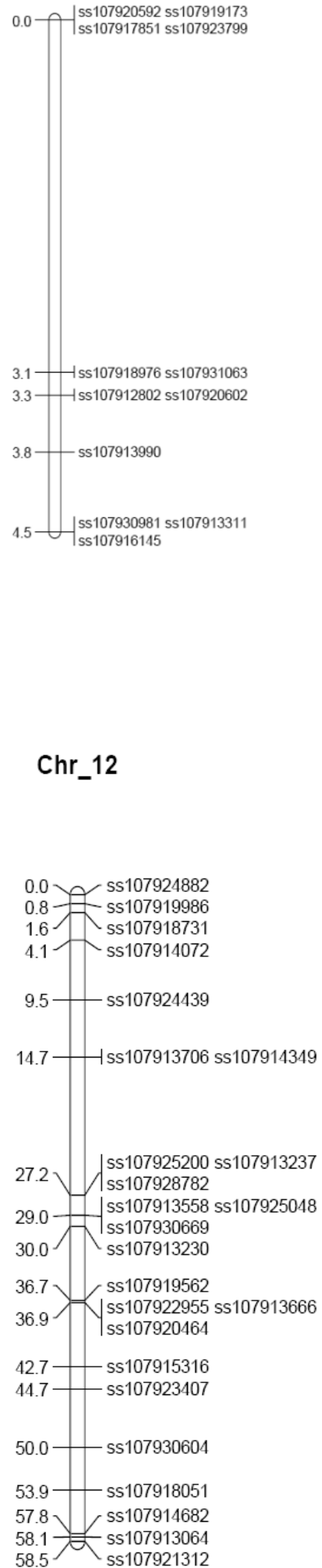

Figure 1. Continued. 
Chr_13a

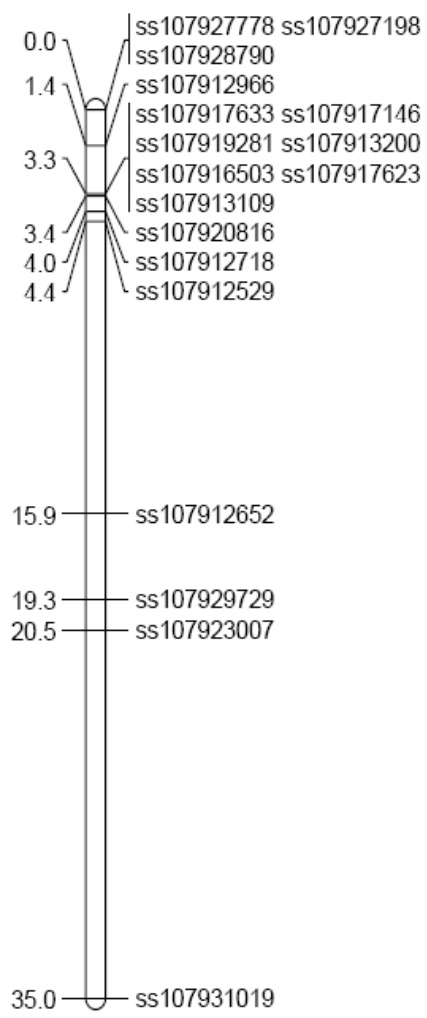

Chr_15b

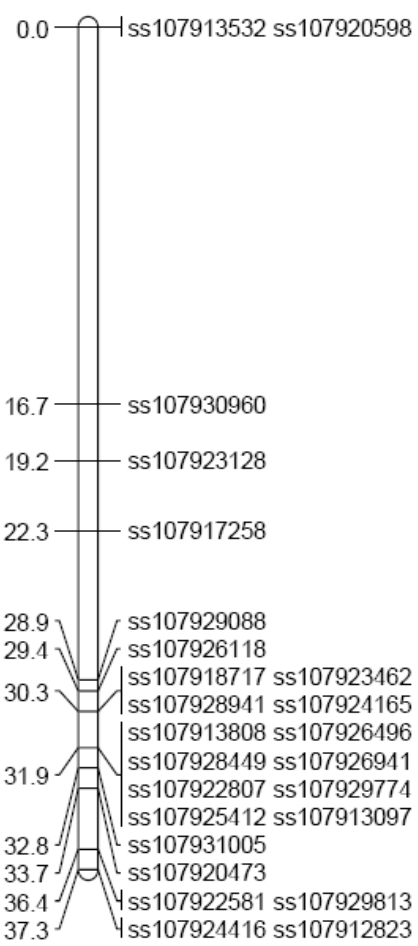

Chr_13b

$11.9-\mathrm{ss} 107920828$

$13.8-\mathrm{ss} 107912991$

$21.9 \_$ss107913481

22.9 ss107924946

26.9- $s s 107918951$

27.9 Yss107924336 ss107920654

\begin{tabular}{l||l}
38.7 & ss 107919342
\end{tabular}

43.5 - $\mathbf{s} 107924542$

51.2- - - ss107929664 ss107912922 52.3 Yss107919896 ss107912665 54.7 - ss 107927651

$61.1 \sqrt{\text { ss107917837 }}$

61.3 $=$ ss107917485 ss107929998

Ss107915606

61.7 ss107920248

$65.9{ }_{\mathrm{s} 107912768}$

\section{Chr_16a}

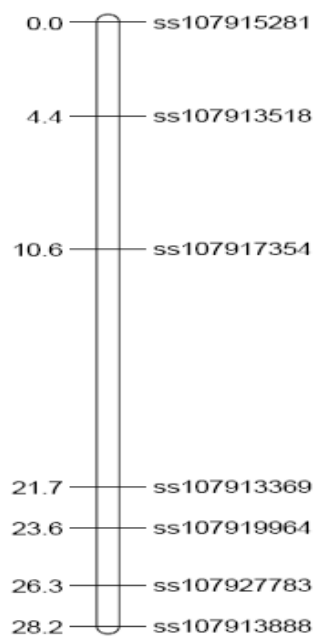

Chr_14

Chr_15a

0.0 لss107926530 ss107928907

1.3 A I ss107913662 ss107912950

3.0 - ss107913527

77 - ss107930533

8.8 $\mathrm{Ns} 107921725$ ss107930438

$8.8 \quad$ ss107922861 ss107913289

21.1 - ss107915148

$30.4-$ Iss107925149 ss107927267

$33.8-$ ss107912772

45.1 ss107913280

Hss107930135 ss107924114

51.8 ss107916335 ss107916333

ss107926137

53.5 ss107926963

53.7 ss107923311

64.4- - ss107912842

73.1 - ss107929660

80.4 ss107914625

81.6 ss107915529

84.1 ss107919846 $_{\text {ss }}$

87.1 S $_{\mathbf{S} 107929807}$

$90.6 \gamma_{\text {ss107912880 }}$

98.5 ss107913657

99.5 $/$ ss107913677 ss107919843 ss107912654

\section{Chr_16b}

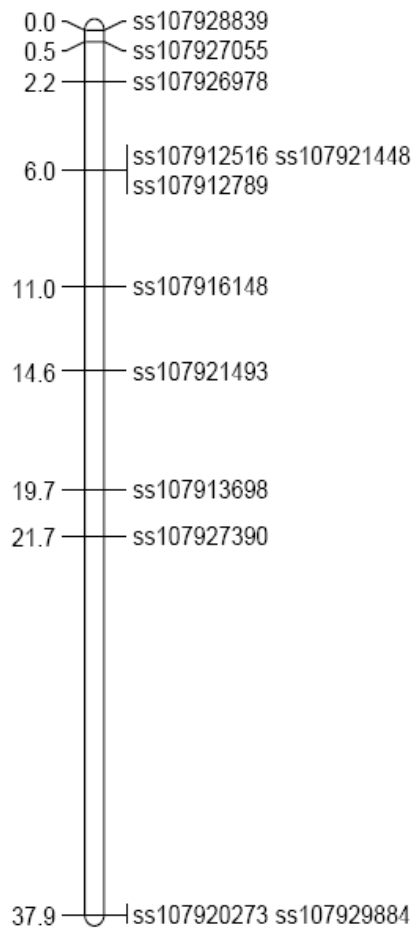

Figure 1. Continued.

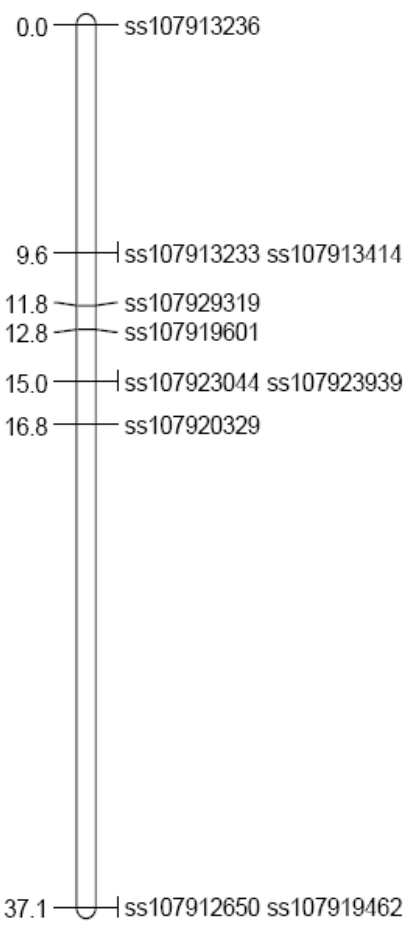

Chr_17a

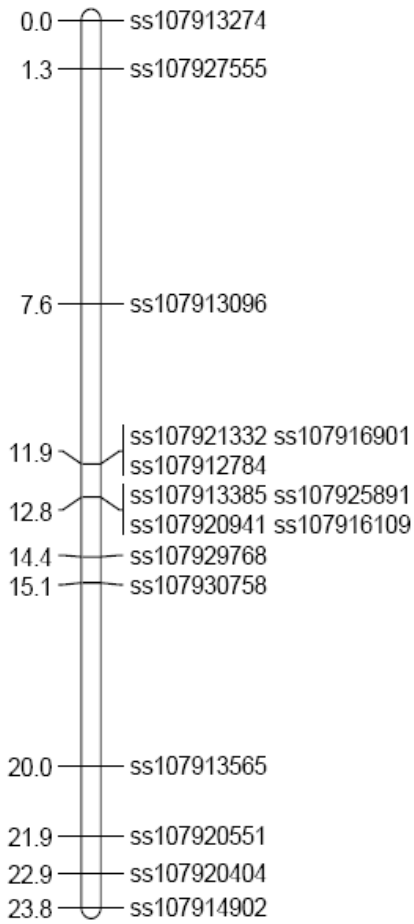


Chr_17b

ss107929993 ss107925594

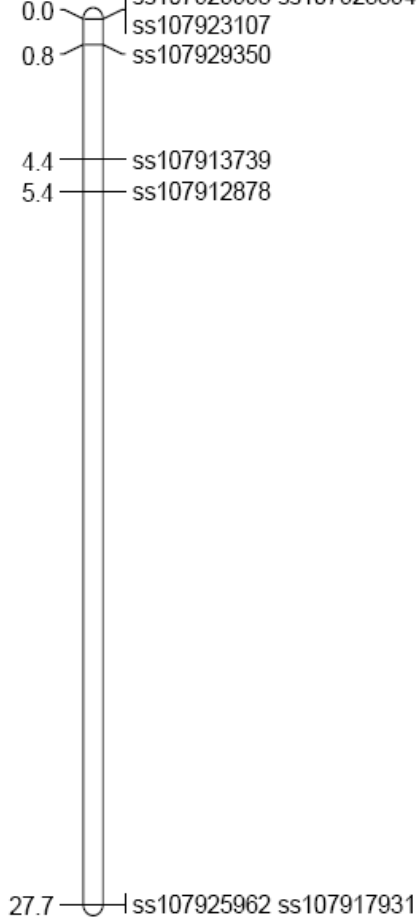

Chr_19

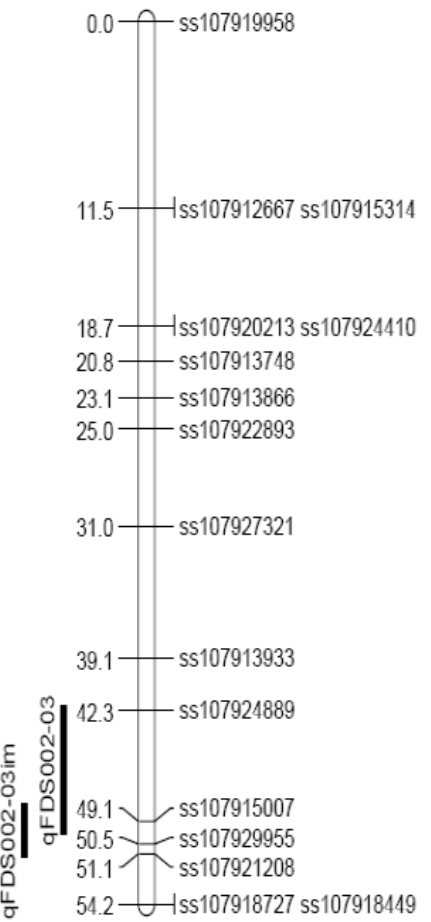

Chr_17c

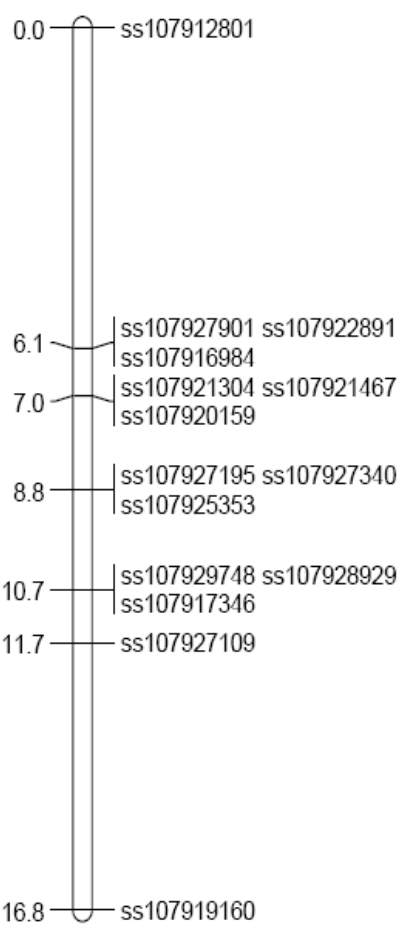

Chr_20a

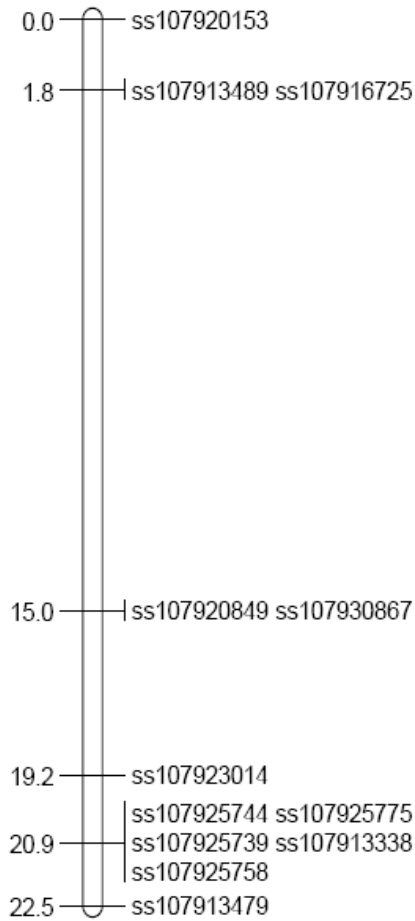

Chr_17d

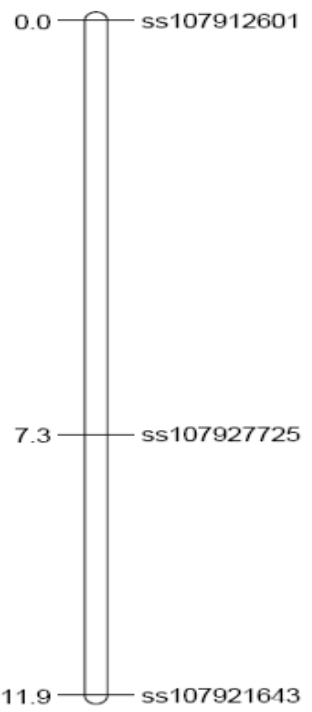

Chr_20b

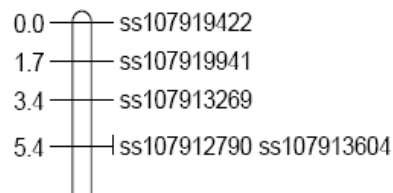

Chr_18

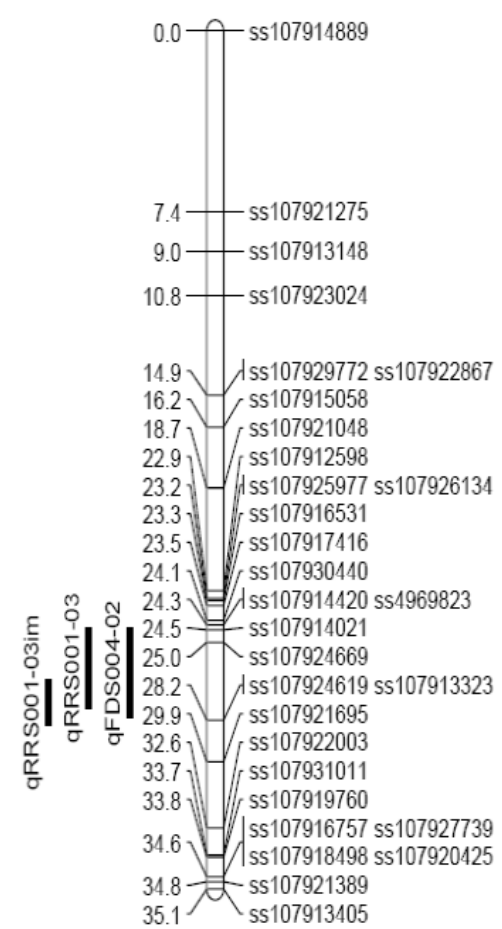

Figure 1. Continued. 
ss107917031-ss107912977 on chromosome 6 (LG C2). The QTL interval spanned approximately $15.9 \mathrm{cM}$, had a peak LOD score of 2.9, and an R2 of $2.4 \%$ (Table 2, Figure 1). The thirteenth QTL that was associated with the FDS 3 trait (qFDSO0306) was identified by the marker interval ss107915722ss107918074 on chromosome 8 (LG A2). The QTL interval spanned approximately $13 \mathrm{cM}$, had a peak LOD score of 2.7, and an R2 of $17.4 \%$ (Table 2, Figure 1). The fourteenth QTL that was associated with the FDS 4 trait (qFDS004-03) was identified by the marker interval ss $107924445-$ ss 107918378 on chromosome 4 (LG C1). The QTL spanned approximately $26.6 \mathrm{cM}$, had a peak LOD score of 2.6 , and an R2 of $4.8 \%$ (Table 2, Figure 1).

QTL that underlie both FDS 3 (qFDSO03-03 and qFDSO0305), and FDS 4 (qFDS004-03 and qFDS004-04) were identified on chromosome 6 (LG C2) (Table 2, Figure 1). Similarly, QTL that underlie both FDS 4 (qFDS004-02) and RRS (qRRS001-03) were identified on chromosome 18 (LG G) (Table 2, Figure 1).

\section{Common Genes within the QTL Regions}

Using CIM, approximately 1,459 genes were identified within the genomic regions containing QTL for FDS 2, FDS 3, FDS 4, and RSS (Table 4-Supplementary data). The most frequently occurring genes ( $>5$ copies) within these regions were those encoding for plant-type serine-threonine protein kinase (Van Bentem et al., 2008), pentatricopeptide repeat-containing protein, MYB-related protein, RNA binding protein, ATP binding cassette transporter, protein phosphatase, ologopeptide transporter, ascorbate oxidase, cytochrome P450, copper transporter protein, CDC2-related kinase, amino acid transporter, zinc finger protein, mitochondrial carrier protein, membrane associated finger protein, homeobox protein, ATP dependent helicase, ATP independent helicase, subtilisin-related serine protease, sensor histidine kinase, RAS-related GTPase, NAD dependent epimerase, glucosyl transferase, F-Box/LRR protein, centurin, aspartyl protease, aquaporin transporter, thioredoxin-related protein, synthaxin, solute carrier protein, and many others (Table 4Supplementary data).

\section{Discussion}

The first 'PI438489' by 'Hamilton' high density SNP-based genetic map presented here is among the few high density maps published in soybean to date (Choi et al., 2007; Hyten et al., 2010b; Vuong et al., 2010) and among the few in crops and other plant species (Feltus et al., 2010). The map can be used to discover new QTL for other agronomic traits and to decipher the candidate genes that underlie these traits.

Several chromosomes are fragmented in to more than one LG $15 a$ and $5 b ; 11 a, 11 b, 11 c$, and $11 d ; 13 a$ and $13 b ; 15 a$ and $15 b ; 16 a$ and $16 b ; 17 a, 17 b, 17 c$, and 17d; $20 a$ and $20 \mathrm{~b})$ which increased the number of LGs into 31 compared to 20 chromosomes may be due to the relatively small population size used in this study (50 RILs). We are in the process of increasing this population size to $>100$ RILs in order to construct a robust genetic linkage map based on this population and to accurately detect QTL for important agronomic traits.

Previously, Kassem et al. (2007) reported nine QTL for SDS resistance. In the same publication, a decade (1996-2006) of SDS resistance QTL mapping in ExF was also summarized. QTL reported in Kassem et al. (2007) were identified using the 'Essex' by 'Forrest' RIL population and the updated ExF genetic map based mainly on SSR markers (Kassem et al., 2006). Later reports increased the number of QTL recognized to 14 (Prabhu et al., 1999; Farias et al., 2007; Kazi et al., 2007, 2008) in diverse germplasm. In the study reported herein, fourteen QTL for SDS resistance were identified, twelve QTL for foliar (FDS) and three QTL for root (RRS) symptoms caused by F. virguliforme infection. At the same position of the QTL (qFDS002-01) identified on chromosome 1 (LG D la), QTL for soybean cyst nematode (SCN) resistance were identified using this same population (Yue et al. 2001). QTL for several other agronomic traits such as javanese root-knot nematode resistance, protein concentration, oil content, and leaf length, were also identified in this region (SoyBase 2010). At the same region of qFDS002-02 identified on chromosome 10 (LG O), only one QTL for Southern root-knot nematode resistance was identified (SoyBase 2011; Li et al., 2001). Similarly, on the same region containing qFDS002-03 identified on chromosome 19 (LG L), QTL for lodging, seed linoleic acid content, plant height, and sucrose concentration were identified and mapped (SoyBase, 2011 ). Interestingly, a QTL for sclerotinia stem rot resistance was identified and mapped $5 \mathrm{cM}$ downstream from this region containing qFDSO02-03. The two QTL qFDS003-01 and qFDS003-02 identified on chromosome 2 (LG D1b) were only $8 \mathrm{cM}$ apart and QTL for several morphological traits such as plant height, yield, leaf width, and leaf length were identified within this region (SoyBase, 2011 ). On chromosome 6 (LG C2), the genomic region containing qFDSO03-03, qFDS003-05, qFDSO04-03, and qFDS004-04 spanned from $17 \mathrm{cM}$ to $40 \mathrm{cM}$ (approx. $23 \mathrm{cM}$ ) and could be designated as one region that underlie resistance to both FDS 3 and FDS 4 . Approximately $10 \mathrm{cM}$ upstream of this region, a QTL for SCN resistance was identified (SoyBase, 2011 ; Wang et al., 2001).

Using the same RIL population (PI 438489BxH), QTL for SCN resistance were mapped $10 \mathrm{cM}$ upstream and $60 \mathrm{cM}$ downstream from the region containing qFDS003-03, qFDS003-05, qFDS004-03, and qFDS004-04 (Yue et al., 2001) on chromosome 6 (LG C2). Approximately $80 \mathrm{cM}$ downstream of this region, QTL for SDS resistance were identified and mapped in different genetic backgrounds (lqbal et al., 2001; Kassem et al., 2006, 2007). Recently, QTL for SDS resistance (cqRfs4; leaf DX) were identified approximately $60 \mathrm{cM}$ from the first region containing qFDSO03-03, qFDSO03-05, qFDSO04-03, and qFDS004-04 (Kazi et al., 2008) on LG C2 which indicates the existence of several SDS resistance QTL on this chromosome. On chromosome 8 (LG A2), a cluster of SDS resistance QTL containing both qFDS003-04 and qRRS001-03 was identified from 2 to $28 \mathrm{cM}$ (26 cM span). Interestingly, a QTL for SCN resistance (Concibido et al., 2004), and SDS resistance (SoyBase, 2011) were identified $4 \mathrm{cM}$, and $20 \mathrm{cM}$ from this region, respectively. On chromosome 11 (LG B 1), QTL for hypocotyls length and aluminum tolerance were identified on the same region containing qFDS004-01 and a cluster of QTL for SCN resistance was iden- 
tified approximately 40 cM from this region (Yue et al., 2001; Concibido et al., 2004). Chromosome 18 (LG G) is of particular interest since it contains a cluster of SDS and SCN resistance QTL identified in different genetic backgrounds (Kassem et al. 2006, 2007; Kazi et al., 2008; Concibido et al., 2004). In this study, the region identified on this chromosome contains both qFDS004-02 and qRRS001-03 which indicates that this region confers resistance to both foliar (FDS) and root (RRS) symptoms. This agreed with earlier results (Meksem et al., 1999; lqbal et al., 2001; Kassem et al., 2006, 2007) was in contrast to what has been previously reported in 'Flyer' by 'Hartwig' RIL population (Kazi et al., 2008).

Interestingly, a QTL for SDS resistance was mapped only 2 CM from the region containing qRRS001-01 identified on chromosome 3 (LG N) (Njiti et al. 2002). On chromosome 4 (LG C1) containing qRRS001-02 and qFDS004-03, no QTL for disease resistance have been mapped in this region; however, QTL for several morphological traits such as seed weight, pod maturity, and seed yield have been mapped at this location (SoyBase, 2011 ).

The most frequently occurring genes within these QTLs were plant-type serine-threonine protein kinase, pentatricopeptide repeat-containing protein and MYB-related protein. The four QTLs collectively had ten copies of each gene. QTL qFDSO0301 had four copies of each of the three genes which is the greatest number of copies. The serine-threonine protein kinase gene is a stress-induced gene which helps plants to cope with environmental stresses such as drought, salinity, and cold (Xinguo et al., 2010). The pentatricopeptide repeat-containing protein is essential for RNA editing in chloroplasts and mitochondrial transcripts (Kotera et al., 2005; Bentolila et al., 2010), and the MYB-related protein is a member of a class of transcription factors identified to be involved in plant stress responses (Martin and Paz-Ares, 1997, Ge et al., 2011 , Uehara et al., 2010).

\section{Conclusions}

In this study, we constructed the first 'PI438489B' by 'Hamilton' SNP-based map and identified fourteen QTL that underlie SDS resistance including both resistance to foliar and root rot symptoms caused by F. virguliforme infection. The map presented here is among the few high density map published in soybean (Choi et al., 2007; Hyten et al. 2010b; Vuong et al., 2010) and other plant species (Feltus et al., 2010). The map can be used to discover new QTL for other agronomic traits and to decipher the candidate genes that underlie these traits.

Seven of the intervals encompassing the QTL had been identified previously (on LGs C1, C2, D 1 b, G, L, N and O) associated with resistance to SDS and this provided strong evidence that the map and trait scoring methods used were valid. Seven QTL were identified in novel locations (LGs A2 (2), B 1, C2, D 1 a, D 1 b and O). Some of these QTL might be confirmed in NILs or by further mapping in a larger portion of the population. Equally some QTL may prove to have been type II errors by additional experiments.

Several studies reported SNP markers associated with QTL for several traits in soybean (Vuong et al., 2007). However, only one of these studies reported an exclusively SNP-based genetic map (Hyten et al., 2010a). However, SNPs are being integrated with SSRs, RFLPs, AFLPs, and other markers to construct high density genetic linkage maps (Hyten et al. 2010a; Hyten et al. $2010 \mathrm{~b}$ ) that are being used by the soybean research community and biotechnology industry to help improve soybean (Vuong et al., 2007). Therefore, the SNP-based map and the QTL regions presented here can be used in breeding programs to develop soybean cultivars with increased resistance to SDS.

\section{List of Abbreviations}

SDS: Sudden death syndrome; SNP: Single nucleotide polymorphism; RFLP: Restriction fragment length polymorphism; AFLP: Amplified fragment length polymorphism; RAPD: Random amplified polymorphic DNA; DAP: Days after planting; RIL: Recombinant inbred line.

\section{Acknowledgments}

The authors would like to thank the United Soybean Board, lowa Soybean Association, North Central Soybean Research Program, and the following individuals for their dedication and contributions to this work: Nieves Rivera, Manuel Mendez, Peter Lundeen and Kyle Vander Molen, lowa State University; Karen Williams for assistance with the GoldenGate assay.

\section{References}

Aoki T, K O'Donnell, and MM Scandiani (2005) Sudden death syndrome of soybean in South America is caused by four species of Fusarium: Fusarium brasiliense sp. nov., F. cuneirostrum sp. nov., F. tucumaniae, and $F$. virguliforme. Mycoscience 46:162-183.

Barbazuk WB, SJ Emrich, HD Chen, L Li, and PS Schnable (2007) SNP discovery via 454 transcriptome sequencing. Plant Journal 51:910918.

Bentolila S, W Knight, and M Hanson (2010) Natural Variation in Arabidopsis Leads to the Identification of REMEl, a Pentatricopeptide Repeat-DYW Protein Controlling the Editing of Mitochondrial Transcripts. Plant Physiology 154:1966-1982.

Brookes AJ (1999) The essence of SNPs. Gene 234:177-186.

Cho RJ, M Mindrinos, DR Richards, RJ Sapolsky, M Anderson, E Drenkard, J Dewdney, TL Reuber, M Stammers, N Federspiel et al. (1999) Genome-wide mapping with biallelic markers in Arabidopsis thaliana. Nat Genet 23:203-207.

Choi IY, DL Hyten, LK Matukumalli, Q Song, JM Chaky, CV Quigley, K Chase, KG Lark, RS Reiter, MS Yoon, EY Hwang, SI Yi, ND Young, RC Shoemaker, CP Van Tassel, JE Specht, and PB Cregan (2007) A soybean transcript map: Gene distribution, haplotype and singlenucleotide polymorphism analysis. Genetics 176: 685-696.

Collins FS, LD Brooks, and A Chakravarti (1998) A DNA Polymorphism Discovery Resource for Research on Human Genetic Variation. Genome Res 8:1229-1231.

Concibido VC, BW Diers, and PR Arelli (2004) A Decade of QTL Mapping for Cyst Nematode Resistance in Soybean. Crop Sci 44:1 $121-$ 1131

Fan JB, A Oliphant, R Shen, BG Kermani, F Garcia, KL Gunderson, M Hansen, F Steemers, SL Butler, and P Deloukas (2003) Highly parallel SNP genotyping, Cold Spring Harbor Symposia on Quantitative 
Biology. Cold Spring Harbor Laboratory Press; 2003.

Farias NAF, R Hashmi, M Schmidt, SR Carlson, GL Hartman, S Li, RL Nelson, and BW Diers (2007) Mapping and confirmation of a new sudden death syndrome resistance QTL on linkage group D2 from the soybean genotypes 'PI 567374' and 'Ripley'. Mol Breeding 20: 53-62.

Feltus FA, J Wan, SR Schulze, JC Estill, N Jiang, and AH Paterson (2010) An SNP Resource for Rice Genetics and Breeding Based on Subspecies Indica and Japonica Genome Alignments. Genome Res 14: $1812-1819$.

Ge Y, Y Li, DK Lv, X Bai, W Ji, H Cai, AX Wang, YM Zhu (2011) Alkaline-stress response in Glycine soja leaf identifies specific transcription factors and ABA-mediated signaling factors. Funct Integr Genomics 11: 369-379.

Hsu CY, C An, S Saha, DP Ma, JN Jenkins, B Scheffler, and DM Stelly (2008) Molecular and SNP characterization of two genome specific transcription factor genes GhMyb8 and GhMybl0 in cotton species. Euphytica 159 (1-2): 259-273.

Hyten DL, SB Cannon, Q Song, N Weeks, EW Fickus, RC Shoemaker, JE Specht, AD Farmer, GD May, and PB Cregan (2010a) Highthroughput SNP discovery through deep resequencing of a reduced representation library to anchor and orient scaffolds in the soybean whole genome sequence. BMC Genomics 11: 38.

Hyten DL, IY Choi, Q Song, JE Specht, TE Carter, RC Shoemaker, EY Hwang, LK Matukumalli, and PB Cregan (2010b) A high density integrated genetic linkage map of soybean and the development of a 1,536 Universal Soy Linkage Panel for QTL mapping. Crop Sci 50:960-968

Hyten DL, Q Song, IY Choi, MS Yoon, JE Specht, LK Matukumalli, RL Nelson, RC Shoemaker, ND Young, and PB Cregan (2008) Highthroughput genotyping with the GoldenGate assay in the complex genome of soybean. Theor Appl Genet 116 (7): 945-952.

Iqbal MJ, K Meksem, VN Niiti, MA Kassem, and DA Lightfoot (2001) Microsatellite markers identity three additional quantitative trait loci for resistance to soybean sudden-death syndrome (SDS) in Essex $x$ Forrest RILs. Theor Appl Genet 102: 187-192

Kassem MA, K Meksem, AJ Wood, and DA Lightfoot (2007) Loci Underlying SDS and SCN Resistance Mapped in the 'Essex' by 'Forrest' Soybean Recombinant Inbred Lines. Rev Biol Biotech 6 (1): 2-10.

Kassem MA, J Shultz, K Meksem, Y Cho, AK Wood, MJ Iqbal, and DA Lightfoot (2006) An updated 'Essex' by 'Forrest' linkage map and composite interval mapping of QTL underlying six soybean traits. Theor Appl Genet 113 (6): 1015-1026.

Kazi S, VN Niiti, TW Doubler, J Yuan, MJ lqbal, S Cianzio, and DA Lightfoot (2007) Registration of the Flyer by Hartwig recombinant inbred line mapping population. J Plant Regis 1: 175-178.

Kazi S, J Shultz, J Afzal, J Johnson, VN Niiti, and DA Lightfoot (2008) Separate loci underlie resistance to root infection and leaf scorch during soybean sudden death syndrome. Theor Appl Genet 116: 967-977.

Keim P, BW Diers, TC Olson, and RC Shoemaker (1990) RFLP mapping in soybean: association between marker loci and variation in quantitative traits. Genet 126: 735-742.

Kotera E, M Tasaka, and T Shikanai (2005) A pentatricopeptide repeat protein is essential for RNA editing in chloroplasts. Nature 433: 326-330.

Lark KG, K Chase, F Adler, LM Mansur, JH Orf (1995) Interactions between quantitative trait loci in soybean in which trait variation at one locus is conditional upon a specific allele at another. PNAS 92: 4656-4660.

Li S, YK Tam, and GL Hartman (2000) Molecular differentiation of Fusarium solani f. sp. glycines from other $F$. solani based on mitochondrial small subunit rDNA sequences. Phytopathology 90: 491-497.
Li Z, L Jakkula, RS Hussey, JP Tamulonis, and HR Boerma (2001) SSR mapping and confirmation of the QTL from PI96354 conditioning soybean resistance to southern root-knot nematode. Theor Appl Genet 103 (8): 1167-1 173.

Lindblad-Toh K, E Winchester, MJ Daly, DG Wang, JN Hirschhorn, JP Laviolette, K Ardlie, DE Reich, E Robinson, P Sklar, N Shah et al. (2000) Large-scale discovery and genotyping of single nucleotide polymorphisms in the mouse. Nat Genet 24 (4): 381-385.

Lu HH, SS Feng, D Kyle, P Stephens, TC Corbin, and J Thompson (2010) Genetic loci associated with Fusarium solani tolerance in soybean. United States Patent 7,767,882.

Luo Y, K Hildebrand, SK Chong, O Myers, and JS Russin (2000) Soybean Yield Loss to Sudden Death Syndrome in Relation to Symptom Expression and Root Colonization by Fusarium solani f. sp. Glycines. Plant Dis 84 (8): 914-920.

Martin C and J Paz-Ares (1997) MYB transcription factors in plants (review). Trends Gen 13 (2): 67-73.

Meksem K, TW Doubler, K Chancharoenchai, VN Njiti, SJC Chang, PR Arelli, PB Cregan, LE Gray, PT Gibson, and Lightfoot (1999) Clustering among loci underlying soybean resistance to Fusarium solani, SDS and SCN in near-isogenic lines. Theor Appl Genet 99: 11311142.

Mohring S, F Salamini, and K Schneider (2004) Multiplexed, linkage group-specific SNP marker sets for rapid genetic mapping and fingerprinting of sugar beet (Beta vulgaris L.). Mol Breed 14: 475-488.

Munkvold GP and JK O'Mara (2002) Laboratory and growth chamber evaluation of fungicidal seed treatments for maize seedling blight caused by Fusarium species. Plant Disease 86 (2): 143-150.

Nickell CD, DJ Thomas, and P Stephens (1990) Registration of 'Hamilton' soybean. Crop Sci 30: 1364.

Nicod JC and CR Largiader (2003) SNPs by AFLP (SBA): A rapid SNP isolation strategy for non-model organisms. Nucl Acids Res 31 (5) e 19: 1-5.

Niiti VN, JE Johnson, TA Torto, LE Gray, and DA Lightfoot (2001) Inoculum rate influences selection for field resistance to sudden death syndrome in the greenhouse. Crop Sci 41: 1726-1731.

Njiti VN, K Meksem, MJ Iqbal, JE Johnson, MA Kassem, KF Zobrist, VY Kilo, and DA Lightfoot (2002) Common loci underlie field resistance to soybean sudden death syndrome in Forrest, Pyramid, Essex, and Douglas. Theor Appl Genet 104: 294-300.

Prabhu RR, VN Niiti, B Bell-Johnson, JE Johnson, ME Schmidt, JH Klein, and DA Lightfoot (1999) Selecting soybean cultivars for dual resistance to soybean cyst nematode and sudden death syndrome using two DNA markers. Crop Sci 39: 982-987.

Schmutz J, SB Cannon, J Schlueter, J Ma, T Mitros, W Nelson, DL Hyten, Q Song, JJ Thelen, J Cheng, D Xu, U Hellsten, GD May, Y Yu, T Sakurai, T Umezawa, MK Bhattacharyya, D Sandhu, B Valliyodan, E Lindquist, M Peto, D Grant, S Shu, D Goodstein, K Barry, M FutrellGriggs, B Abernathy, J Du, Z Tian, L Zhu, N Gill, T Joshi, M Libault, A Sethuraman, XC Zhang, K Shinozaki, HT Nguyen, RA Wing, PB Cregan, J Specht, J Grimwood, D Rokhsar, G Stacey, RC Shoemaker, and SA Jackson (2010) Genome sequence of the palaeopolyploid soybean. Nature 463: 178-183.

Shoemaker RC and JE Specht (1995) Integration of the Soybean Molecular and Classical Genetic Linkage Groups. Crop Sci 35: 436446.

Song QJ, LF Marek, RC Shoemaker, KG Lark, VC Concibido, X Delannay, JE Specht, and PB Cregan (2004) A new integrated genetic linkage map of the soybean. Theor Appl Genet 109:122-128.

SoyBase, 2011 [http://soybase.agron.iastate.edu/]. Accessed December, 2011.

Uehara T, S Sugiyama, H Matsuura, T Arie, and C Masuta (2010) Resistant and susceptible responses in tomato to cyst nematode are 
differentially regulated by salicylic acid. Plant Cell Physiol 51 (9): 1524-36.

Van Bentem FS, D Anrather, I Dohnal, E Roitinger, E Csaszar, J Joore, J Buijnink, A Carreri, C Forzani, ZJ Lorkovic, A Barta, D Lecourieux, A Verhounig, C Jonak, and H Hirt (2008) Site-specific phosphorylation profiling of Arabidopsis proteins by mass spectrometry and peptide chip analysis. J Proteome Res 7 (6): 2458-2470.

Vuong TD, DA Sleper, JG Shannon, and HT Nguyen (2010) Novel quantitative trait loci for broad-based resistance to soybean cyst nematode (Heterodera glycines Ichinohe) in soybean PI 567516C. Theor Appl Genet 121 (7): 1253-1266.

Vuong TD, X Wu, MDS Pathan, B Valliodan, HT Nguyen (2007) Genomics Approaches to Soybean Improvements. In: Genomics-Assisted Crop Improvement. Volume 2. Edited by RK Varshney, R Tuberosa, pp. 243-279.

Wang D, BW Diers, PR Arelli, and RC Shoemaker (2001) Loci underly- ing resistance to Race 3 of soybean cyst nematode in Glycine soja plant introduction 468916. Theor Appl Genet 103 (4): 561-566.

Wang S, CJ Basten, and ZB Zeng (2005) Windows QTL Cartographer 2.5. Department of Statistics, NCSU, Raleigh, NC, [http://statgen. ncsu.edu/qtlcart/WQTLCart.htm].

Wu SB, I Tavassolian, G Rabiei, P Hunt, M Wirthensohn, JP Gibson, CM Ford, and M Sedgley (2009) Mapping SNP-anchored genes using high-resolution melting analysis in almond. Mol Genet and Gen 282 (3): 273-281.

Xinguo M, Z Hongying, T Shanjun, C Xiaoping, and J Ruilian (2010) TaSnRK2.4, an SNF1-type serine/threonine protein kinase of wheat (Triticum aestivum L.), confers enhanced multistress tolerance in Arabidopsis. J Exp Bot 61 (3): 683-696.

Yue P, PR Arelli, and DA Sleper (2001) Molecular characterization of resistance to Heterodera glycines in soybean 'PI 438489B'. Theor Appl Genet 102: 921-928. 